\title{
Unusual presentation of breast lump- diagnosed on FNAC as tubercular lesion
}

\author{
Sengupta $S,{ }^{1}$ Mondal RK, ${ }^{1^{*}}$ Ray $R,{ }^{1}$ Bose $K,{ }^{1}$ Ghosh $\mathrm{TK}^{1}$
}

${ }^{1}$ Dept of Pathology, Bankura Sammillani Medical College, Bankura, West Bengal, India

\author{
* Corresponding Author: \\ Dr. Rajib Kumar Mondal \\ Assistant Professor, \\ Dept of Pathology, \\ Vill-Barjora, Schooldanga, \\ Po+ps-Barjora, Dist-Bankura, \\ West Bengal ,Pin-722202, India \\ Email-rajibkmondal@yahoo.co.in \\ Citation \\ Sengupta S, Mondal RK, Ray R, Bose K, Ghosh TK. \\ Unusual presentation of breast lump-diagnosed on \\ FNAC as tubercular lesion. Nepal Journal of Medical \\ Sciences 2013;2(2):187-9.
}

\begin{abstract}
Tuberculosis of breast is a rare disease even in endemic areas accounting for $0.1 \%-0.5 \%$ of all infections. Multiparous young females are commonly affected particularly during pregnancy and lactation.

In the present study, we are reporting a case of tuberculosis of breast with ipsilateral axillary involvement in a multiparous young non lactating female. Diagnosis was confirmed after cytological evaluation.

Clinical or radiological distinction of mammary tuberculosis from breast carcinoma or abscess is often difficult to achieve. Cytology is an effective method for confirmation of these uncommon disorder an also can reduce unnecessary surgery which should be restricted for complicated cases.
\end{abstract}

Keywords: Breast; FNAC; tuberculosis

\section{Introduction:}

Tuberculosis remains as one of the leading causes of morbidity and mortality from infectious etiology both in the developing as well as develop countries. Extra pulmonary involvement of almost any organ or tissue is reported in nearly $17.9 \%$ of tuberculosis infections. Breast along with spleen and skeletal muscles are considered to be maximally resistant to tubercular infections. ${ }^{1-3}$ Mammary glands are unsuitable sites for survival and multiplication of mycobacterium tuberculosis and reported incidence of involvement varies from $0.5 \%$ of all tuberculosis cases. ${ }^{2,4}$ But the incidence of breast tuberculosis is increasing worldwide due to increase in number of geriatric population, emergence of drug resistance strain of bacilli and immunosuppressant (Breast lesions can be the presenting manifestation of HIV infection). ${ }^{1,2,5,6}$

We are reporting a case of mammary tuberculosis, diagnosed by aspiration cytology to stress inclusion of this rare lesion as a possible differential diagnosis during assessment of breast aspirate.

Case report: A 31 yrs female with a left breast lump was referred to pathology department of Bankura Sammillani Medical College (BSMC), Bankura, West Bengal, India for FNAC of the lesion. The patient was in apparently good health and had four children but was non lactating. Physical examination revealed a moderately large sized $(6 \mathrm{~cm}$ in diameter), hard, tender, mobile swelling with irregular borders and redness of overlying skin situated at the upper and outer quadrant of left breast for one month and two firm mobile lymph nodes in left axilla, approximately $2 \mathrm{~cm}$ in diameter each. A clinical diagnosis of pyogenic lesions/? Inflammatory carcinoma. The haematological reports were within normal limits except neutrophilic leucocytosis. Blood sugar and other related biochemical reports were within normal limits. Chest X-Ray was within normal limits.

Aspirates yielded thick pus like material. Prepared smears from both sites demonstrated caseating necrosis, epithelioid granuloma (fig-1), Langhan's type of giant cells 
and lymphocytes during microscopical evaluations. Z-N stained smears showed presence of acid fast bacilli(Pauci bacillary) (fig-2). A diagnosis of mammary tuberculosis with involvement of axillary lymph node was given. Subsequently patient was treated with standard antituberculosis drug rising with complete recovery of both axillary and breast lesions.

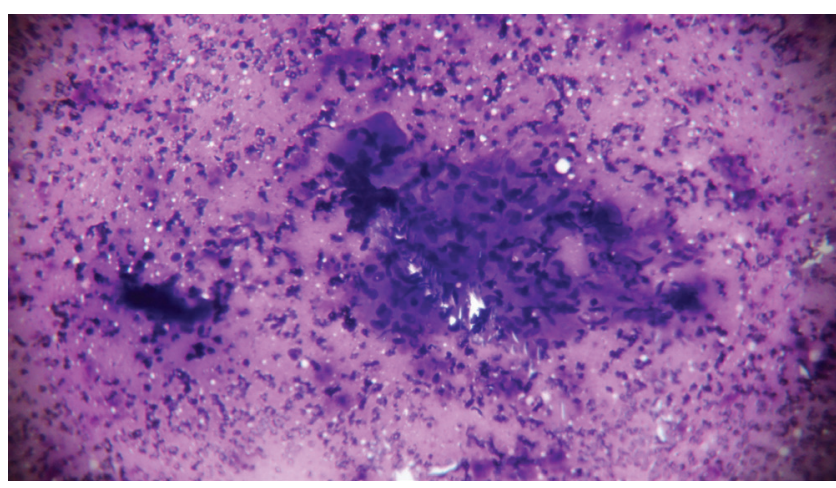

Fig-1 Photo micrograph showing epitheliod granuloma with caseating necrosis (x 400, Leishman- Giemsa)

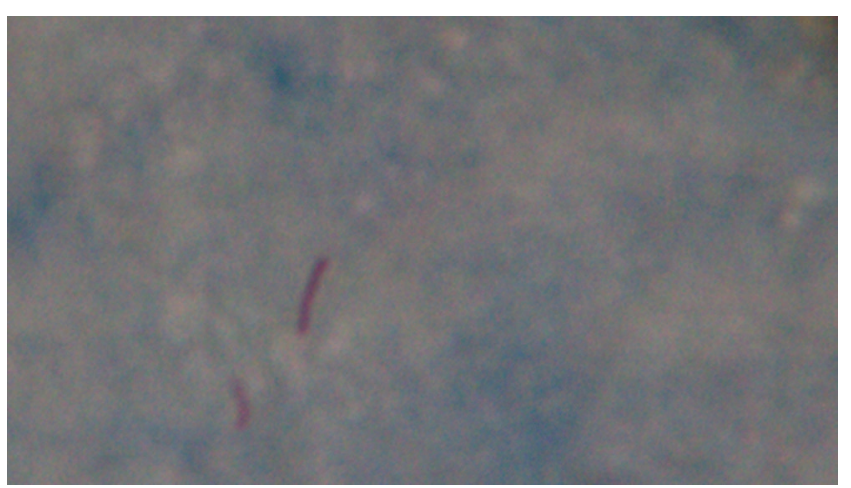

Fig-2:Photo micrograph showing AFB (x 1000 Z.N. Stain)

Discussion: Tuberculosis is a rare disease of breast with incidence ranging from $0.025 \%$ to $0.1 \%$ in developed countries and up to $0.3 \%$ to $5 \%$ in endemic region. ${ }^{1,2}$ Majority of reported cases involved young multiparous females of 20 to 40 yrs age group owing to constant physiological changes of the gland in this age group with increased susceptibility to trauma and infection. ${ }^{1,2,7,8}$ Pregnancy and lactation further enhance chance of acquiring infection due to increased vascularity and dilation of ducts predisposing to trauma. ${ }^{2,7}$ Male breast can also be affected though infrequently. ${ }^{2,5,6}$ Present patient under discussion was young, multiparous and non lactating.

Breast tuberculosis can be primary or secondary. Primary involvement is rarely reported where only location of the disease is breast. ${ }^{8}$ Infection is acquired by hematogenous route or by direct extention of contact infected material with irritated skin or breast ducts particularly during lactation. Secondary infection can reach breast by blood, lymphatic, or directly from the lung, pleura, mediastinum or articular lesions. ${ }^{2,7,8}$ Centripetal lymphatic spread was the most accepted avenue for spread of infections. Ipsilateral cervical or axillary lymph nodes are involved in majority (50\%-70\%) of cases suggesting breast involvement by retrograde lymphatic extension. ${ }^{2,6,8}$ In the present case breast involvement occurred due to secondary ipsilateral axillary lymph node lesions.

Clinical manifestations of breast lesions are highly variable. Constitutional symptoms like fever weight loss, anorexia, night sweats are often absent as in the present case. ${ }^{1}$ Commonly breast lesions are nodular with or without ulceration and sinus formation and pain less lump.

Tuberculosis of breast usually occurs in upper and outer quadrant of breast is more commonly involved owing to proximity to the axillary lymph nodes, quite similar to our experience. Other presentation includes diffuse nodularity, abscess formation and multiple sinuses. Multinodularity or bilaterality is rarely reported. ${ }^{1,2}$ Fixity and skin changes resembling carcinoma can be present. ${ }^{1,7}$

Radiological investigations like USG and mammography are unreliable in distinguishing breast tuberculosis from carcinoma. CT scan though not confirmatory can detect extension of the lesion and differentiate primary from secondary lesions. ${ }^{1,10}$

FNAC is the cornerstone for correct diagnosis of breast tuberculosis. ${ }^{6}$ Demonstration of caseating necrosis with epithelioid granuloma is currently accepted as the diagnostic findings during cytological evaluation leading to definite diagnosis in up to $73 \%$ cases with appropriate clinical presentation. ${ }^{1,2,6}$

Attempts must be done for demonstration of acid fast bacilli by staining of culture though in vain in majority of cases. ${ }^{1,2}$ However failure to demonstrate AFB and even necrosis on cytology does not exclude diagnosis of breast tuberculosis. Because of small quantity of sample examined. ${ }^{11}$ Nucleic acid amplification test (NAATS) like PCR can detect Mycobacterium tuberculosis complex DNA. $\mathrm{PCR}$ is recommended in culture in negative cases though its sensitivity also low particularly in AFB smear negative cases. Open biopsy has to be performed in few cases and still regarded as the most reliable method for diagnosis of breast tuberculosis. ${ }^{13}$

Apart from carcinoma, differential diagnosis of breast tuberculosis includes few rare diseases like traumatic fat 
necrosis, plasma cell mastitis, chronic pyogenic abscess, mammary dysplasia, fibroadenoma, granulomatous mastitis,sarcoidosis or fungal granulomas. Identification of breast tuberculosis does not exclude concomitant breast cancer as both condition can occasionally co exist. ${ }^{1,11,13}$

Management of breast lesions is essentially medical with complete resolution in most of the cases. FNAC should be repeated after completion of therapy to ascertain that the residual mass is fibrotic. Surgery is rarely needed in complicated refractory cases. ${ }^{1,13}$

\section{Conclusion:}

Breast tuberculosis is an uncommon lesion which cannot be differentiated clinically or radiologically from breast cancer and abscess. FNAC is accepted as the most preferred method for diagnosis though histopathological confirmation is required. We are presenting the present case to stress the inclusion of this rare disorder in the list of possible differential diagnosis during cyto evaluation of breast lump and to give the message that simple AFB staining of the breast aspirate can resolve the problem

\section{References:}

1. Kao PT, Tu MY, Tang SH. Tuberculosis of the breast with erythema nodosum : a case report. journal of medical case reports 2010;4:124.

2. Murat K, Mehmet T, Sule B, et al. Tuberculosis of breast. Eur J Gen Med 2010;7:216-9.

3. Madhusudhan KS, Gamanagatti S: Primary breast tuberculosis masquerading as carcinoma. Singapore Med J 2008;49:e3.

4. Mahasweri V, Tyagi SP, Asraf SM, et al. Tubercular mastitis: A clinicopathological study. Ind J Surg 1993;55:129-33.

5. Maroulis I, Spyropoulos C, Vasiliki Z, et al. Mammary tuberculosis mimicking breast cancer: a case report. J Med Case Reports 2008;2:1752-3.

6. Silva BB, Lopes-Costa PV, Pires CG, et al. Tuberculosis of the breast: analysis of 20 cases and a literature review. Trans R Soc Trop Med Hyg 2009;103:559-63.

7. Maroulis I, Spyropoulos C, Zolota V, et al. Mammary tuberculosis mimicking breast cancer: a case report. J Med Case Reports 2008;2:34.

8. Selim K, Resat K, Ayhan O, et al. Primary Tuberculosis of breast. Diagn Interv Radiol 2005;11:210-2.

9. Zandrino F, Monetti F, Gandolfo N. Primary tuberculosis of the breast. A case report. Acta Radiologica 2000;41:61-3.

10. Khanna R, Prasanna GV, Gupta P, et al: Mammary tuberculosis: report on 52 cases. Postgrad Med J 2002;78:422-4.

11. Tewari M, Shukla HS: Breast tuberculosis: diagnosis, clinical features and management. Indian J Med Res 2005;122:103-10.

12. Shi-ping LUH, Jeng-dong HSU, Yih-shyong LAI, et al. Primary tuberculous infection of breast: experiences of surgical resection for aged patients and review of literature. J Zhejiang Univ Sci B 2007;8:580-3.

13. Shinde SR, Chandawarkar RY, Deshmukh SP: Tuberculosis of the breast masquerading as carcinoma: a study of 100 patients. World J Surg 1995;19:379-81. 